\title{
Potential neutrino signals from galactic $\gamma$-ray sources
}

\author{
A Kappes ${ }^{1}$, J Hinton ${ }^{2,3}$, C Stegmann ${ }^{1}$ and F A Aharonian ${ }^{2}$ \\ ${ }^{1}$ Physics Institute, University Erlangen-Nuremberg, Erwin-Rommel-Str. 1, D-91058 Erlangen, \\ Germany \\ ${ }^{2}$ Max-Planck-Institut für Kernphysik, Saupfercheckweg 1, D-69117 Heidelberg, Germany \\ ${ }^{3}$ Landessternwarte, Königstuhl, D-69117 Heidelberg, Germany \\ E-mail: kappes@physik.uni-erlangen.de
}

\begin{abstract}
Recent measurements of the energy spectra of Galactic $\mathrm{TeV} \gamma$-ray sources with the H.E.S.S. instrument have been used to calculate the expected high energy neutrino fluxes from these sources. Based on the results the expected event rates in a next generation neutrino telescope in the Mediterranean Sea, KM3NeT, have been estimated, assuming an instrumented volume of $1 \mathrm{~km}^{3}$. We find that for energies above $1 \mathrm{TeV}$ event rates of a few neutrinos per year can be expected from the brightest $\gamma$-ray sources. Although these rates are comparable to those of the background from atmospheric neutrinos a detection of individual sources seems possible.
\end{abstract}

\section{Introduction}

Even a century after its discovery the origin of the cosmic radiation (CR) remains to be a mystery. A smoking gun evidence for the location of CR acceleration sites would be the detection of $\mathrm{TeV}$ cosmic neutrinos. However, even after several years of intense search no high energy cosmic neutrino has been identified yet.

High energy cosmic neutrinos are produced in reactions of accelerated protons or nuclei with the ambient interstellar gas through the decay of charged pions or kaons. On the other hand, neutral pions, also generated in these reactions, decay into photons resulting in a high energy $\gamma$-ray flux. Hence, sources of $\mathrm{TeV} \gamma$-ray emission represent prime targets for neutrino telescopes.

The recent progress in Galactic $\gamma$-ray astronomy using the H.E.S.S. instruments provides for the first time a comprehensive list of bright Galactic $\mathrm{TeV} \gamma$-ray sources for which the neutrino flux can be estimated. Most of these sources lie on the Southern Hemisphere and are therefore best visible to detectors on the Northern Hemisphere. Here, we consider a neutrino telescope in the Mediterranean Sea, KM3NeT [2], with an instrumented volume of $1 \mathrm{~km}^{3}$.

Table 1 contains the results of our calculations for a number of selected sources, where a more detailed description of this work together with the event rates for all current H.E.S.S. Galactic $\gamma$-ray sources can be found in [3].

\section{Galactic $\gamma$-ray emission}

The Galactic $\gamma$-ray sources observed by H.E.S.S. can be assigned to 4 different classes, where for each class a hadronic origin is more or less likely:

Supernova Remnants (SNRs): Young SNRs are considered as the best candidates for CR acceleration. In total, H.E.S.S. has observed 5 SNRs where two of them, RX J1713.9-3946 and RX J0852.0-4622, are young and very bright. Their detailed study allows the accurate 


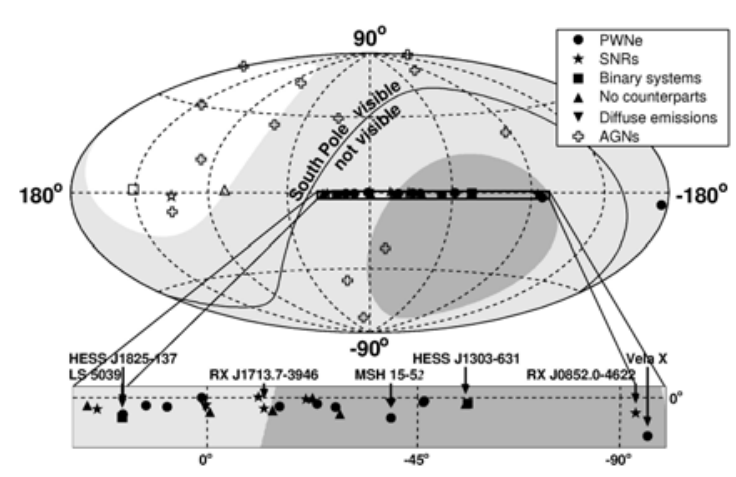

Figure 1. Sky map of $\mathrm{TeV} \gamma$-ray sources in Galactic coordinates together with their visibility to neutrino telescopes. The shaded regions represent $25-75 \%$ (light grey) and $>75 \%$ (dark grey) visibility to a detector in the central Mediterranean Sea for energies below $\sim 100 \mathrm{TeV}$. The solid curve shows the extent of the visibility of a South Pole detector (IceCube).

prediction of the expected neutrino fluxes in the most relevant energy band between $0.1 \mathrm{TeV}$ and $100 \mathrm{TeV}$.

Binary Systems (Binary): Two objects in this class have been detected by H.E.S.S., LS 5039 and PSR B1259-63. In general, these objects are treated as leptonic (inverse Compton) accelerators but in the case of LS 5039 also a hadronic interpretation of the $\gamma$-ray emission exist [4].

No counterpart (NCP): The lack of any good counterpart at other wavelengths supports the hadronic acceleration interpretation and renders these sources good candidates for neutrino production.

Pulsar Wind Nebulae (PWNe): As in the case of Binary Systems the observed $\gamma$-ray emission is generally interpreted in terms of leptonic acceleration. However, again hadronic interpretations exist as for example in the case of Vela X [5].

Figure 1 shows a sky map of all currently known $\mathrm{TeV} \gamma$-ray sources.

\section{Neutrino fluxes}

For our calculations we use recent parameterisations of the pion and secondary particle production in hadronic interactions [6] to derive the relationship between $\gamma$-ray and neutrino spectra in the case of the primary proton spectrum given by:

$$
\frac{\mathrm{d} N_{p}}{\mathrm{~d} E_{p}}=k_{p}\left(\frac{E_{p}}{1 \mathrm{TeV}}\right)^{-\alpha} \exp \left(-\frac{E_{p}}{\epsilon_{p}}\right) .
$$

Assuming full mixing we find that the neutrino and $\gamma$-ray spectra at Earth can be well described by:

$$
\frac{\mathrm{d} N_{\gamma / \nu}}{\mathrm{d} E_{\gamma / \nu}} \approx k_{\gamma / \nu}\left(\frac{E_{\gamma / \nu}}{1 \mathrm{TeV}}\right)^{-\Gamma_{\gamma / \nu}} \exp \left(-\sqrt{\frac{E_{\gamma / \nu}}{\epsilon_{\gamma / \nu}}}\right) .
$$

The relations between the different parameters are given by: $k_{\nu} \approx(0.71-0.16 \alpha) k_{\gamma}, \Gamma_{\nu} \approx \Gamma_{\gamma} \approx$ $\alpha-0.1$ and $\epsilon_{\nu} \approx 0.59 \epsilon_{\gamma} \approx \epsilon_{p} / 40$, . Figure 2 shows as example the neutrino spectrum of the SNR RX J1713.9-3946. The error bands of the neutrino and $\gamma$-ray spectrum include the $1 \sigma$ systematic uncertainties based on the systematic uncertainties of the published H.E.S.S. spectra.

The calculation of the predicted neutrino flux is based on some essential assumptions where the validity of each assumption has to be assessed on a source-by-source basis. The most important assumptions are:

- No significant contribution of non-hadronic processes to the measured $\gamma$-ray signal; 


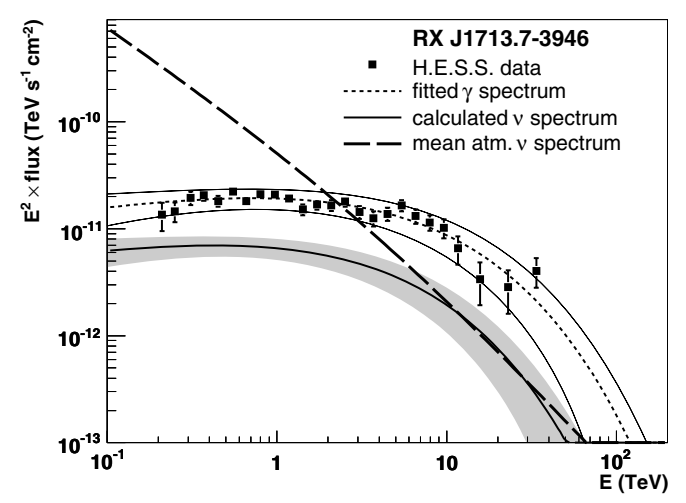

Figure 2. The measured $\gamma$-ray flux and the estimated neutrino flux with their error bands together with the atmospheric neutrino flux. The atmospheric neutrino flux is integrated over the search window and averaged over one day.

- No significant $\gamma$-ray absorption within the source, i.e. radiation and matter densities are sufficiently low for most $\gamma \mathrm{s}$ to escape;

- No significant $p \gamma$ interaction (radiation density low);

- Charged pions decay before interacting (matter density is low);

- Muons decay without significant energy loss (magnetic field is low);

- Nucleus-nucleus interactions produce pion spectra which are similar enough to the $p p$ case that they can be treated in the same way;

- The size of the emitting region within each source is large enough that oscillations will produce a fully mixed neutrino signal at the Earth $\left(\nu_{e}: \nu_{\mu}: \nu_{\tau}=1: 1: 1\right)$.

\section{Neutrino event rates}

Source rate: Given a neutrino spectrum $\mathrm{d} N_{\nu} / \mathrm{d} E_{\nu}$ at the Earth from a source the event rate in a neutrino telescope can be calculated as:

$$
\frac{\mathrm{d} N_{\nu}}{\mathrm{d} t}=\int \mathrm{d} E_{\nu} A_{\nu}^{\text {eff }} \frac{\mathrm{d} N_{\nu}}{\mathrm{d} E_{\nu}} .
$$

Here, $A_{\nu}^{\text {eff }}$ is the neutrino effective area of the detector comprising the neutrino attenuation in the Earth as well as the neutrino conversion probably into a muon and the muon detection efficiency. Figure 3 displays the estimated effective area for muon neutrinos of a future KM3NeT detector with an instrumented volume of $1 \mathrm{~km}^{3}[7]^{1}$. As an example Fig. 4 shows the integrated number of events in 5 years for energies $>E_{\nu}$ for RX J1713.9-3946. Table 1 lists the calculated ranges of number of detected neutrinos for all H.E.S.S. sources with an observed cut-off in the $\gamma$-ray spectrum.

Background rate: Neutrinos produced in hadronic interaction of charged CR on the opposite side of the Earth are indistinguishable from those of cosmic origin. The calculation of the corresponding event rate is performed in the same way as for the cosmic neutrinos, using the optimal search window for an extended source of about $\Theta_{\text {opt }}=1.6 \times \sqrt{\sigma_{\mathrm{PSF}}^{2}+\sigma_{\mathrm{src}}^{2}}$. Here, $\sigma_{\text {src }}$ is the radius of the source and $\sigma_{\mathrm{PSF}}=0.3$ the point spread function of the detector. For the atmospheric neutrino flux the parameterisation of Volkova [8] is used. The corresponding integrated daily flux from the sky region of RX J1713.9-3946 is shown in Fig. 4.

\footnotetext{
1 Since currently dedicated reconstruction software for KM3NeT is not available yet, its effective area is calculated requiring signals from the muon in at least 10 photo sensors.
} 


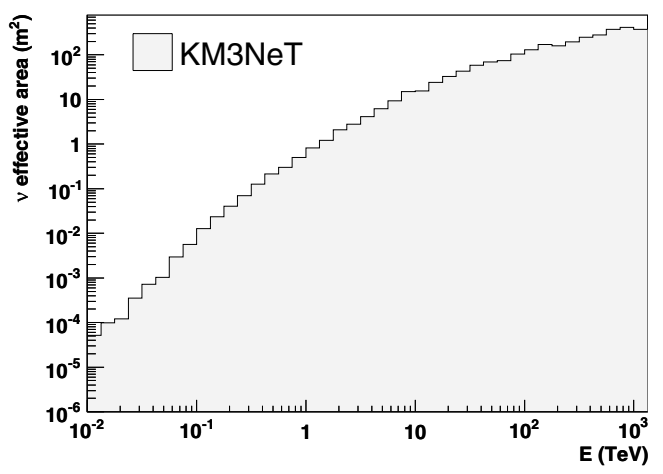

Figure 3. Effective area $A_{\nu}^{\text {eff }}\left(E_{\nu}\right)$ for muon neutrinos of the KM3NeT detector with an instrumented volume of $1 \mathrm{~km}^{3}$.

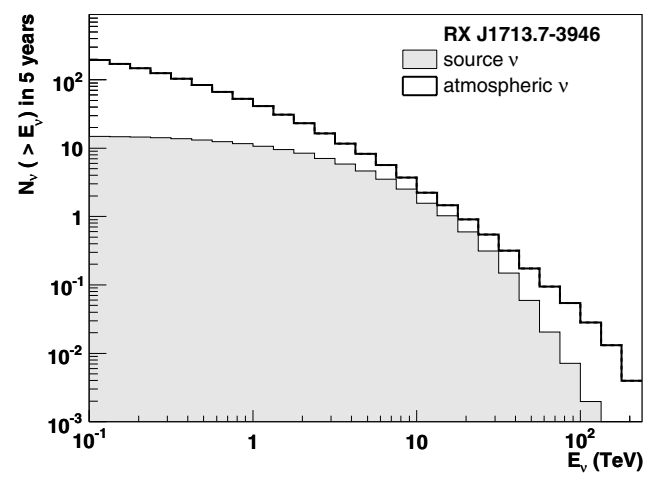

Figure 4. Number of detected events in 5 years with neutrino energies $>E_{\nu}$ for RX J1713.7-3946 in the KM3NeT detector.

Table 1. Selected sources from the H.E.S.S. catalogue with range of estimated neutrino event rates within the search window in KM3NeT for 5 years of operation for neutrino energies above $1 \mathrm{TeV}$ and $5 \mathrm{TeV}$. Also shown is the expected atmospheric neutrino background. The values under Dia. represent the diameter of the source in $\gamma$-rays.

\begin{tabular}{lccrrrr}
\hline \multirow{2}{*}{ Name } & & \multicolumn{4}{c}{$E_{\nu}>1 \mathrm{TeV}$} & \multicolumn{2}{c}{$E_{\nu}>5 \mathrm{TeV}$} \\
& Type & Dia. $\left(^{\circ}\right)$ & Source & Bkg & Source & Bkg \\
\hline Vela X & PWN & 0.8 & $9-23$ & 23 & $5-15$ & 4.6 \\
RX J0852.0-4622 & SNR & 2.0 & $7-15$ & 104 & $1.9-6.5$ & 21 \\
RX J1713.7-3946 & SNR & 1.3 & $7-14$ & 41 & $2.6-6.7$ & 8.2 \\
HESS J1825-137 & PWN & 0.5 & $5-10$ & 9.3 & $2.2-5.2$ & 1.8 \\
Crab Nebula & PWN & $<0.1$ & $4.0-7.6$ & 2.0 & $1.1-7.2$ & 1.1 \\
HESS J1303-631 & NCP & 0.3 & $0.8-2.3$ & 11 & $0.1-0.5$ & 2.1 \\
LS 5039 (INFC) $\dagger$ & Binary & 0.1 & $0.3-0.7$ & 2.5 & $0.1-0.3$ & 0.5 \\
\hline
\end{tabular}

$\dagger$ Assuming no $\gamma$-ray absorption within source. INFC specifies the phase of inferior conjunction of the binary system as defined in [9].

\section{Discussion}

The brightest $\gamma$-ray sources produce neutrino rates above $1 \mathrm{TeV}$ comparable to the background from atmospheric neutrinos. For KM3NeT with an instrumented volume of $1 \mathrm{~km}^{3}$ a few cosmic neutrinos per year from each of these sources can be expected and the detection of individual sources seems possible. However, due to low statistics of source neutrinos the detection of $\mathrm{TeV}$ signals from a major fraction of H.E.S.S. sources will be difficult for $1 \mathrm{~km}^{3}$ class neutrino telescopes.

[1] Hinton J A 2004 New Astron. Rev. 48331

[2] Katz U F 2006 Preprint astro-ph/0606068

[3] Kappes A, Hinton J A, Stegmann C and Aharonian F A 2006 Preprint astro-ph/0607286

[4] Aharonian F A, Anchordoqui L A, Khangulyan D and Montaruli T 2006 J. Phys. Conf. Series 39408

[5] Horns D et al 2006 A $\& A 451$ L51

[6] Kelner S R, Aharonian F A and Bugayov V V 2006 Phys. Rev. D74 034018

[7] Kuch S 2005 Preprint astro-ph/0606507

[8] Volkova L V 1980 Sov. J. Nucl. Phys. 31(6) 784

[9] H.E.S.S. Coll. Aharonian F A et al 2006 Preprint astro-ph/0607192 